\title{
Eine ungarische Debatte aus dem Jahr 1926 über die Funktion der Volksabstimmung im politischen System

\author{
Hintergrund, Standpunkte und die spätere Entwicklung
}

\section{A Hungarian Debate from 1926 about the role of referendums in the political system \\ Background, standpoints and the later development}

In 1926, the publicist Hugó Ignotus (1869-1949) and the sociologist Róbert Braun (1879-1937) debated in the Hungarian journal "Nyugat" the possible and desirable roles of referendums in the political system. This debate was unique because institutions of direct democracy were neither regulated nor practiced in Hungary at that time; not even in-depth scientific or political discussions took place about the issue. Ignotus was of the opinion that referendums could - at least partly - take over the role of the parliament and be used as a controlling power against authoritarian leaders. He proposed to enable citizens to directly elect and dismiss the political leader and to lay down the guidelines for the government in referendums; the government should only elaborate and implement detailed regulations. Braun argued in his response that institutions of direct democracy cannot replace the representative power and that citizens should not be overburdened by too frequent referendums. Direct democracy may work as a corrective to the parliament but it is not suitable for preventing authoritarian tendencies. He also emphasized the importance of political maturity as a prerequisite for a convenient use of referendums and popular initiatives. Later developments justified Braun's opinion. The article presents the standpoints of the discussion partners and places their views in the context of preceding and subsequent evolution.

Keywords: direct democracy-Hungary - interwar period - plebiscite - popular initiative - referendum

\section{Einführung}

Die Debatte, die im Jahr 1926 zwischen dem Publizisten Hugó Veigelsberg (1869-1949) und dem Soziologen Róbert Braun (1879-1937) in der ungarischen Zeitschrift „Nyugat“ (,West“) veröffentlicht wurde, ist eine der sehr wenigen Schriften, die sich in der Zwischenkriegszeit in Ungarn mit der Funktion der Volksabstimmung und anderen direktdemokratischen Institutionen beschäftigten. Moderne Instrumente der direkten Demokratie erschienen erstmals in der zweiten Hälfte des 18. Jh. in den ehemaligen britischen Kolonien in Neuengland, ${ }^{1}$ tauchten dann in den französischen Verfassungen der bürgerlichen Revolution auf ${ }^{2}$ und wurden im 19. Jh. auch in der Schweiz, zuerst in den Kantonen, dann auch auf Bundesebene, eingeführt und weiterentwickelt. ${ }^{3}$ Nach dem Ersten Welt-

\footnotetext{
${ }^{1}$ LOBINGIER, The People's Law 163-203; AuER, Le référendum 71-76.

2 FREI, Direkte Demokratie 4-12.

${ }^{3}$ VATTER, Kantonale Demokratien 228-240; DERS., Direkte Demokratie 72-77.
} 
krieg kam es zu ihrer ersten größeren Verbreitung, als in vielen neuen Demokratien, wie der Weimarer Republik, der Republik Österreich, den Baltischen Staaten und im Irischen Freistaat verschiedene Arten von Referenden, Volksinitiativen und Plebisziten in die neuen Verfassungen aufgenommen wurden. ${ }^{4} \mathrm{Zu}$ einer ähnlichen Entwicklung kam es in Ungarn nicht, obwohl die Idee der direkten Machtausübung des Volkes seit der Französischen Revolution auch in Ungarn bekannt war. ${ }^{5}$ Die ungarische Elite war aber solchen Ideen gegenüber sehr zurückhaltend. ${ }^{6}$ Als die bürgerliche Volksvertretung im Jahre 1848 eingeführt wurde, wurden das Repräsentativprinzip und das freie Mandat der Abgeordneten unbestreitbar in der ungarischen Gesetzgebung. Weder mit dem österreichungarischen Ausgleich im Jahre 1867 noch mit dem Gesetzesartikel Nr. I. von 1920 wurde das geändert. Verschiedene Kräfte der politischen Opposition strebten vor allem nach der Erweiterung des Wahlrechts und nicht nach direkter Demokratie. Nur die Ungarische Sozialdemokratische Partei und die Bürgerliche Radikale Partei nahmen „die direkte Volksgesetzgebung auf dem Wege der Initiative und des Referendums" in ihre Parteiprogramme in den Jahren $1903^{7}$ bzw. $1914^{8}$ als Forderung auf. Die politi-

\footnotetext{
${ }^{4}$ KOMÁROMI, Parliaments 58-60.

${ }^{5}$ Siehe z.B. den im Jahre 1793 verfassten Verfassungsentwurf von Ignác Martinovics, der direktdemokratische Elemente enthielt (veröffentlicht in: BENDA, A magyar jakobinus mozgalom 897-908).

${ }^{6}$ Siehe z.B. die Aussage des ungarischen Publizisten, László Szalay, aus dem Jahr 1844: SzALAY, Diéta 204205, in englischer Übersetzung zitiert von KOMÁROMI, Popular Rights 3.

${ }^{7}$ ERÉNYI, A magyar munkásmozgalom 140. Die Verfasser des Programms der Ungarischen Sozialdemokratischen Partei waren wahrscheinlich vom Eisenacher Programm der Deutschen Sozialdemokratischen Arbeiterpartei (1869) inspiriert, die ebenfalls die „Einführung der direkten Gesetzgebung (das heißt Vorschlags- und Verwerfungsrecht) durch das Volk" vorsah, siehe MOMMSEN, Parteiprogramme 312.

${ }^{8}$ PAJKOSSY, Magyarország története 884 .
}

sche Realität war von diesen direktdemokratischen Forderungen weit entfernt. Weder wurden tiefgehende wissenschaftliche Diskurse über das Thema geführt, noch hat sich damit die populärwissenschaftliche Literatur beschäftigt. In diesem Zusammenhang ist die publizistische Debatte aus dem Jahr 1926 ein interessanter Ausnahmefall. In der folgenden Untersuchung werden zuerst die Teilnehmer dieser Debatte vorgestellt, dann ihre Standpunkte im internationalen Kontext präsentiert, schließlich die späteren Entwicklungstendenzen kurz geschildert, damit die Stichhaltigkeit der Argumente der Diskussionspartner beurteilt werden kann.

\section{Die Disputanten}

Hugo Veigelsberg, geboren im Jahre 1869 in Pest, war ein ungarischer Dichter, Schriftsteller, Literaturkritiker und Publizist. Er schrieb unter dem literarischen Pseudonym „Ignotus“. Er war Mitgründer und zwanzig Jahre lang erster Chefredakteur der Zeitschrift "Nyugat", die zwischen 1908 und 1941 als wichtigstes Organ für progressive Dichter und Schriftsteller galt. Eine ganze Epoche der ungarischen Literaturgeschichte wurde nach dieser Zeitschrift benannt. Ignotus studierte Rechtswissenschaft in Budapest. Nach seinem Studium arbeitete er als Journalist und schrieb Berichte aus Deutschland, aus der Türkei, dem Balkan und aus den USA für die Tageszeitung „Magyar Hírlap“. 1919 emigrierte er nach Wien, nahm aber auch von dort aktiv am ungarischen Literaturleben teil. 1938 flüchtete er wegen den Judengesetzen nach London und 1941 in die USA. Erst 1948 kehrte er schwerkrank nach Budapest zurück, wo er ein Jahr später gestorben ist. ${ }^{9}$

Róbert Braun, geboren im Jahre 1879 in Arad, war ein ungarischer Soziologe, Bibliothekar,

\footnotetext{
${ }^{9}$ KENYERES, Életrajzi lexikon, „Ignotus, Veigelsberg Hugó“; HegedÜs, Arcképcsarnok, „Ignotus“.
} 
Dorfforscher und Lexikograph. Er studierte in Budapest und Paris, ab 1907 unterrichtete er an der Handelsfachmittelschule in Siebenbürgen, in Neumarkt am Mieresch [Marosvásárhely, Targu Mures]. 1911 kehrte er nach Budapest zurück und arbeitete als Vizedirektor der Stadtbibliothek bis 1918. Nach der Räterepublik von 1919 wurde er ins Gefängnis geworfen. Später arbeitete er als Lektor, Übersetzer und Redakteur bei verschiedenen Zeitschriften. Er publizierte über soziologische und wirtschaftliche Themen und übersetzte unter anderem die Werke des US-amerikanischen Ökonomen Henry George (1839-1897) und die über die US-amerikanische Demokratie geschriebenen Teile der zweibändigen Monographie ${ }^{10}$ des britischen Juristen, Historikers und Politikers James Bryce (1838-1922). ${ }^{11}$

Weder Ignotus, noch Braun war ein Fachexperte im Bereich Demokratiegeschichte, direkter Demokratie, Rechts- oder Politikwissenschaften. Aber beide waren Männer mit einer hohen Ausbildung, die sich für gesellschaftliche und politische Probleme interessierten und im Allgemeinen auch überdurchschnittlich informiert waren, nicht nur bezüglich Ungarn, sondern auch über die ausländischen, internationalen Ereignisse und Entwicklungstendenzen. Auch ihre Debatte fokussierte vor allem nicht auf Ungarn, sondern auf den internationalen Kontext.

\section{Der Kontext der Debatte}

Dieser Kontext war der Folgende: Im Laufe des 19. Jh. entstanden in den meisten europäischen Staaten die Grundlagen der demokratischen Entwicklung und der parlamentarischen Regierungsform. Die Parlamente wurden aufgrund des allgemeinen Wahlrechts gewählt und die Verfahren, wodurch die Regierungen zur Verantwortung gezogen werden konnten, waren

\footnotetext{
${ }^{10}$ BRYCE, Modern Democracies.

${ }^{11}$ KENYERES, Életrajzi lexikon, „Braun Róbert“.
}

festgelegt. Das Funktionieren der Parlamente warf jedoch auch Probleme auf. Obstruktion, das Vertreten von Partikularinteressen, Beeinflussung durch Großindustrie und Finanzkapital - um nur einige Probleme zu nennen. Außerdem waren die Parlamentsparteien in vielen Ländern nur kaum disziplinierte Klubs, auf welche man eine stabile parlamentarische Regierung nicht stützen konnte. Besonders dieser letzte Faktor, die politische Instabilität der Regierungen, die häufigen Regierungswechsel, wurden nach dem Ersten Weltkrieg sehr deutlich. Die neuen Demokratien gerieten in eine schwierige Lage. ${ }^{12}$ Töne, die eine stärkere Regierung bzw. eine einflussreichere Exekutivgewalt forderten, wurden immer lauter. Dementsprechend begann die Erosion der neuen, nach dem Ersten Weltkrieg gegründeten Demokratien. Die wirtschaftliche und die damit verbundene politische Krise hat diese Entwicklung beschleunigt. Die Macht der Regierungen bzw. Staatsoberhäupter wurde erweitert, die Kompetenzen des Parlaments eingeschränkt. Die Forderung nach einem starken Führer war aber immer noch gut vernehmbar und das Schlimmste in dieser Hinsicht - die Umformung der demokratischen Ordnungen in totalitäre Regime - ließ noch auf sich warten. Im Jahre 1926 waren nur die ersten Zeichen, z.B. in Italien, zu sehen.

\section{Standpunkte und Argumente}

Manche Aspekte dieses Themenkomplexes wurden von Ignotus in seinem Artikel mit dem Titel „Der große Schrei nach dem Diktator" ${ }^{13}$ erörtert. In diesem Artikel stellte Ignotus fest, dass die Politik, die Exekutive von Natur aus zur Führung geeignete Politiker brauchte, die ihren Willen auch auf andere Menschen übertragen können. Wenn solche begabten Politiker nicht

\footnotetext{
${ }^{12}$ Siehe dazu GusY, Demokratie in der Krise.

${ }^{13}$ IGNOTUS, A nagy sikoly.
} 
$\mathrm{zu}$ finden sind oder nicht von selbst auftreten, taucht der Wunsch nach einem Diktator, besonders in schweren Zeiten auf. Das Problem der Parlamente ist, sagte Ignotus, dass sie gerade gegen Autokraten als Garantie erfunden wurden, aber mit den Volksvertretungen nur solche politischen Führer erfolgreich zusammenarbeiten können, die die Gabe der Autokraten - den anderen $\mathrm{zu}$ diktieren - haben. Sonst unterliegt der politische Führer im Kampf mit dem Parlament und kann seine Ziele nicht verwirklichen. Wie, mit welchem Instrument könnte man die Vorzüge der Autokratie zur Geltung bringen und zur selben Zeit auch die Demokratie aufrechterhalten? Die Parlamente scheinen dazu nicht geeignet $\mathrm{zu}$ sein. Ignotus fand dieses Instrument in der Volksabstimmung und meinte, dass ein starker politischer Führer, der vom Volk gewählt und abgewählt werden kann, auf der einen Seite, und das Volk, das die wichtigsten Fragen direkt entscheidet, auf der anderen Seite, zusammen eine gute Kombination bilden könnten. Richtlinien für die Politik der Regierung sollten mittels Volksabstimmung gegeben werden; die Detailfragen zu entscheiden und die Durchführung der Beschlüsse gehören zur Kompetenz des politischen Führers und seiner Regierung. Ignotus meinte auch, dass die Elemente dieser Konzeption bereits vorhanden waren: der US-Präsident, einerseits, regiert unabhängig vom Kongress, fast wie ein Diktator; die in England praktizierte häufige Parlamentsauflösung vor unvorhersehbaren oder grundlegenden Entscheidungen, andererseits, wirkt wie eine Volksabstimmung. Diese zwei Komponenten solle man miteinander verknüpfen.

Die Grundidee dieses Lösungsvorschlags führte Ignotus bereits 23 Jahre früher, 1903, in einer längeren Studie in der Zeitschrift „Huszadik Század“ („Zwanzigstes Jahrhundert") aus. In diesem Artikel, der unter dem Titel „Die zu- künftige Gesetzgebung“14 erschienen ist, untersuchte Ignotus verschiedene Krisensymptome des Parlamentarismus: die Unbrauchbarkeit der parlamentarischen Verfahren, mit denen man die Regierung zur Rechenschaft ziehen sollte; der Einfluss verschiedener Interessengruppen auf den Entscheidungsprozess; die Gehaltlosigkeit der parlamentarischen Debatten und der Niedergang des Stils der Parlamentsreden; die Obstruktion; die Schwierigkeiten der Kommunikation zwischen Abgeordneten der verschiedenen Nationalitäten und die Instabilität der von der Laune der Parteien abhängigen Regierung. Er kam zum Schluss, dass die Parlamente den Volkswillen nicht richtig repräsentieren können und dass das auf Vertretung basierende Gesetzgebungsmodell durch die direkte Volksgesetzgebung abgelöst werden soll. Parlamente können nur eine Vorbereitungs-, Deliberationsund Beratungsfunktion erfüllen. Lokale Fragen solle man in den verschiedenen territorialen Autonomien - so Ignotus - ebenfalls durch Volksabstimmung entscheiden.

Róbert Braun kritisierte in seiner Antwort (,,Oligarchie in der Demokratie ${ }^{\prime 15}$ ) den ersterwähnten Artikel von Ignotus vor allem im Hinblick darauf, dass Ignotus politische Führerschaft und Diktatur miteinander vermischt. Es sei gar nicht egal, meinte Braun, ob der Machtinhaber seine Politik im Einklang mit dem Volkswillen oder davon unabhängig durchführte, ob er gewaltsam oder auf gütlichem Wege regierte. Braun wies auch darauf hin, dass nicht nur Diktaturen, sondern auch die meisten Demokratien oligarchische (elitäre) Züge aufweisen. Es sei nicht schwer, sagte er, nicht organisierte Volksmassen gegen ihre eigenen Interessen $\mathrm{zu}$ führen. Eine solche Politik hat aber auch ihre Grenzen. Die Volksabstimmung ersetzt nicht einmal in jenen Ländern das Parlament, wo direkte Demokratie

\footnotetext{
14 VeIGelsberG, A jövendő törvényhozás.

${ }^{15}$ BRAUN, Oligarchia.
} 
häufig praktiziert wird, wie in der Schweiz, in den westlichen Gliedstaaten der USA und in Australien. So eine Rolle wird von der Volksabstimmung auch nicht erwartet. Braun warnte davor, direktdemokratische Instrumente ohne Berücksichtigung der verschiedenen kulturellen und politischen Verhältnisse in andere Staaten umzusetzen. Er war auch in der Frage mit Ignotus nicht einig, welche Faktoren den Wunsch nach dem Diktator hervorrufen. In den lateinischen Staaten trugen dazu die schwache demokratische Kultur, der Mangel an Selbstverwaltung und das Fehlen der großen Parteien auch bei. Also, die Krise war - so Braun - nicht generell der Institution des Parlaments zuzuschreiben.

Ignotus machte in seiner Replik (,Diktatur und Volksabstimmung ${ }^{\prime 16}$ ) klar, dass er nicht für die Diktatur plädierte, sondern nur die Antwort auf die Frage suchte, wie man den Menschen, die heute Diktatur fordern, eine wirksame Regierung ohne die Nachteile der Diktatur (wie z.B. die Ausgeliefertheit an die Willkürherrschaft) gewähren kann. Er wiederholte seinen Standpunkt, dass das Parlament, trotz wiederkehrender Reformversuche, nur eine Kommission unter anderen Machtfaktoren (wie Presse, Interessenverbände und sonstige Institutionen) sei, und dass die Volksabstimmung die Rolle des Parlaments als letztentscheidende Instanz in der Gesetzgebung übernehmen könne. Wenn das von der Volksabstimmung nicht erwartet wird, was kann man dann von diesem Instrument erwarten? - fragte abschließend Ignotus.

Braun stellte in seiner Antwort ("Gesetzgebung ohne Parlament ${ }^{\prime \prime} 17$ ) die zwei wichtigsten Institutionen der modernen direkten Demokratie dar. Das Referendum, mit dem ein vom Parlament bereits verabschiedetes Gesetz vom Volk bestätigt oder verworfen werden kann, und die Volksinitiative, wodurch die Initiatoren mittels

\footnotetext{
${ }^{16}$ IGNOTUS, Diktatúra.

17 BRAUN, Parlament.
}

Unterschriftssammlung über ihren eigenen Gesetzesentwurf eine Volksabstimmung herbeiführen können und so die Gesetzgebung auch ohne die Willensäußerung des Parlaments möglich ist. Diese zwei Instrumente - fügt Braun hinzu ersetzen jedoch nirgendwo das Parlament. Im parlamentarischen Regierungssystem, wo das Parlament den Willen des Stimmvolks repräsentiert, kann die Volksabstimmung als ein technisches Korrektiv des Parlaments funktionieren: wenn eine neue Frage auf die politische Tagesordnung gesetzt wird, worüber sich die Stimmbürgerschaft bei den letzten Parlamentswahlen nicht äußern konnte, können die Stimmbürger auch ohne Parlamentswahlen gefragt werden. In so einem Fall wird die Auflösung des Parlaments politisch nicht nötig sein, sondern das Stimmvolk entscheidet direkt über die Frage. Das Referendum kann auch gegen die Interessendurchsetzung einer Minderheit in der Regierungsmehrheit eingesetzt werden, wenn diese Minderheit die Mehrheit mit dem Austritt aus der Regierungskoalition erpresst. Falls die Mehrheit - unter dem Druck der Minderheit ein Gesetz verabschiedet, kann man nachher gegen dieses Gesetz mittels Referendum ein Volksveto erheben. Diese und ähnliche Verbesserungen bedeuten jedoch keine wesentliche Umgestaltung des parlamentarischen Systems. Dort, wo sie eingeführt wurden, wie in der Schweiz und in den US-Gliedstaaten, werden diese Instrumente seltener benutzt oder nehmen an den Volksabstimmungen weniger Stimmbürger teil, als am Anfang. Braun wies schließlich darauf hin, dass die Volksabstimmung eine viel größere politische Reife erfordert als der reine Parlamentarismus. Wo diese Reife fehlt, wird das Instrument von einem Demagogen missbraucht, wie es unter der Herrschaft von Napoleon III. in Frankreich passierte.

Soweit die Debatte. Auf den zweiten Artikel von Braun kam keine Antwort mehr von Ignotus. Die Diskussion zwischen den Autoren fand kein Echo in politischen Kreisen oder Zeitungen. 


\section{Spätere Entwicklung und Schlussfolgerungen}

Inwieweit bestätigte die spätere Entwicklung die Standpunkte der beiden Publizisten? In der direkten Nachfolgezeit wurden Initiativen und Referenden in manchen neuen Demokratien benutzt, wie z.B. in der Weimarer Republik, wo 1926 („Fürstenenteignung“) und 1929 (,YoungPlan") zwei landesweite Volksabstimmungen abgehalten wurden, die aber wegen des hohen Teilnahmequorums ungültig waren. ${ }^{18}$ Extremistische Kräfte, auch die NSDAP hielten die Volksinitiative für ein attraktives Instrument, wodurch sie ihre Popularität verstärken wollten. Nach Hitlers Machtübernahme haben die Nazis von unten kommende Volksinitiativen und Referendumsverfahren nicht mehr erlaubt, die Volksabstimmung wurde eine politische Waffe der Regierung, ganz wie in der napoleonischen Ära in Frankreich. ${ }^{19}$ Ein zweites Beispiel ist Estland, wo die Regierungsmehrheit die politische Instabilität durch Verfassungsänderungen bekämpfen wollte. Diese Versuche scheiterten aber am Instrument des obligatorischen Verfassungsreferendums, da das Stimmvolk die Verfassungsänderungen nicht guthieß. Schließlich konnte die Veteranenliga, eine rechtsextremistische Gruppe, ihren eigenen Verfassungsentwurf mittels Volksinitiative durchsetzen. Diese neue Verfassung erweiterte die Machtkompetenzen des Staatsoberhauptes und beschränkte drastisch die Macht des Parlaments. Diese Ereignisse ebneten aber letztendlich den Weg für Konstantin Päts, der die Veteranenliga auflöste und ein autoritäres Regime einführte. ${ }^{20}$ Auf Grund dieser Entwicklungen lässt sich feststellen, dass direktdemokratische Institutionen, ohne Tradi-

\footnotetext{
${ }^{18}$ Siehe dazu SCHIFFERS, Elemente 212-215, 231-233; DERS., Weimarer Erfahrungen 55-56, 59-60.

${ }^{19}$ Siehe dazu JUNG, Plebiszit und Diktatur; DERS., Volksabstimmungen.

${ }^{20}$ KOMÁrOMI, Parliaments 71-74.
}

tion und geeignete politische Kultur, auch gefährlich wirken können, insbesondere in Zeiten politischer und wirtschaftlicher Krise. Es stellte sich auch heraus, dass die Volksabstimmung an sich kein wirksames Instrument gegen den Diktator ist, vielmehr besteht die Gefahr, dass ein autoritäres Regime von oben angeordnete Volksabstimmungen für seine eigenen Machtzwecke missbraucht. Róbert Brauns Warnung war in dieser Hinsicht zutreffend.

Nach dem Zweiten Weltkrieg wurden direktdemokratische Instrumente auch in anderen Staaten, oft mit Vorsicht, eingeführt. In Italien z.B. dauerte es bis in die 70er Jahre, dass die direktdemokratischen Instrumente der Verfassung von 1947 implementiert und ihre Benutzung begonnen wurde. ${ }^{21}$ Nach dem politischen Systemwandel in Ostmitteleuropa wurden verschiedene Institutionen auch in diesen Ländern, auch in Ungarn eingeführt. ${ }^{22}$ Die Parlamente bestehen und funktionieren aber auch weiterhin. Direktdemokratische Prozesse können das Parlament - im Gegensatz zur Erwartung des Ignotus - nicht ersetzen. Auch in der Schweiz wird über nicht mehr als $7 \%$ aller Parlamentsvorlagen ein fakultatives Referendum durchgeführt, etwa $93 \%$ der parlamentarischen Gesetzesentwürfe werden ohne direkte Einflussnahme des Volkes verabschiedet. ${ }^{23}$ Dabei spielt auch eine Rolle, was Róbert Braun kurz erwähnt hat: die Belastbarkeit der Stimmbürgerschaft ist begrenzt. Alle Gesetze kann man nicht der Volksabstimmung unterbreiten. Auch heute nicht, wenn die Informationen auf verschiedenen Wegen der Kommunikation technisch einfacher und schneller erreichbar sind als vor etwa 100 Jahren. Ignotus war in dieser Hinsicht zu opti-

\footnotetext{
${ }^{21}$ Siehe dazu CAPRETTI, Direkte Demokratie in Italien 158-163.

22 Über ihre Regelung und Praxis siehe AUER, BÜTZER, Direct Democracy; MARCZEWSKA-RYTKO, Handbook; DIES., Civic Participation.

${ }^{23}$ LINDER, Direkte Demokratie 119.
} 
mistisch. Dann bleiben aber die Institutionen direkter Demokratie, und das sieht man auch in der heutigen Praxis, höchstens Korrektive des Parlaments - wie das Braun bereits 1926 feststellte. In den meisten Fragen hat die spätere Entwicklung seinen Standpunkt bestätigt.

\section{Korrespondenz:}

Dr. László KOMÁrOMI, Budapest

Katholische Péter Pázmány Universität

Szentkirályi u. 28-30.

$\mathrm{H}-1088$ Budapest

komaromi.laszlo@jak.ppke.hu

ORCID-Nr. 0000-0002-2381-3755

\section{Abkürzungen:}

Siehe das allgemeine Abkürzungsverzeichnis: [http://www.rechtsgeschichte.at/media/abk.pdf]

\section{Literatur:}

Andreas AUER, Le référendum et l'initiative populaires aux Etats-Unis (Bâle-Francfort-sur-le-Main 1989).

DERS., Michael BüTZER (Hgg.), Direct Democracy. The Eastern and Central European Experience (Aldershot 2001).

Kálmán BENDA (Hg.), A magyar jakobinus mozgalom iratai (= A magyar jakobinusok iratai Bd. 1, Budapest 1957).

Róbert BRAUN, Oligarchia a demokráciában, in: Nyugat $19(1926 / 16) 322-324$.

DERS., Parlament nélküli törvényhozás, in: Nyugat 19 (1926/22) 794.

James BRYCE, Modern Democracies, 2 Bde. (New York 1921).

Anna CAPRETTI, Direkte Demokratie in Italien, in: Hermann K. HeußnER, Otmar Jung (Hgg.), Mehr direkte Demokratie wagen. Volksentscheid und Bürgerentscheid: Geschichte / Praxis / Vorschläge (München 22009) 157-177.

Tibor ERÉNYI u.a. (Hgg.), A magyar munkásmozgalom a 20. század első éveiben és az 1905-1907-es forradalmi válság idején (Budapest 1955).

Christoph FREI, Direkte Demokratie in Frankreich: Wegmarken einer schwierigen Tradition (=Vorträge am Liechtenstein-Institut, Kleine Schriften 22, Vaduz 1995).

Christoph Gusy (Hg.), Demokratie in der Krise. Europa in der Zwischenkriegszeit (Baden-Baden 2009).

Géza Hegedüs (Hg.), A magyar irodalom arcképcsarnoka (Budapest 1976)

Hugó IGNOTUS, A nagy sikoly a diktátor után, in: Nyugat 19 (1926/15) 229-232

DERs., Diktatúra és népszavazás, in: Nyugat 19 (1926/17) 405-406.

Otmar JuNG, Die Volksabstimmungen der Nationalsozialisten, in: Hermann K. HeUßnER, Otmar JUNG (Hgg.), Mehr direkte Demokratie wagen. Volksentscheid und Bürgerentscheid: Geschichte / Praxis / Vorschläge (München 22009) 91-102.

DERS., Plebiszit und Diktatur: die Volksabstimmungen der Nationalsozialisten. Die Fälle »Austritt aus dem Völkerbund « (1933), "Staatsoberhaupt« (1934) und »Anschluß Österreichs« (1938) (= Beiträge zur Rechtsgeschichte des 20. Jahrhunderts 13, Tübingen 1995).

Ágnes KENYERES (Hg.), Magyar életrajzi lexikon (Budapest 1967) [https://www.arcanum.hu/hu/onlinekiadvanyok/Lexikonok-magyar-eletrajzi-lexikon7428D] (24. 1. 2020). 
László KOMÁROMI, Parliaments and Popular Lawmaking. The German and Estonian Experience in the Interwar Years, in: Parliaments, Estates and Representation 34 (2014/1) 55-75.

DERS,, Popular Rights in Hungary. A Brief Overview of Ideas, Institutions and Practice from the Late 18th Century until Our Days, in: C2D Working Paper Series 35 (2010).

Wolf LINDER, Direkte Demokratie, in: Ulrich KLÖTI u.a. (Hgg.), Handbuch der Schweizer Politik (Zürich ${ }^{21999)}$ 109-130.

Charles Sumner LOBINGIER, The People's Law or Popular Participation in Law-making from Ancient Folk-moot to Modern Referendum (New York 1909).

Maria MARCZewSKA-RYTKO u.a. (Hgg.), Civic Participation in the Visegrad Group Countries after 1989 (Lublin 2018).

Maria MARCZEWSKA-RYTKO (Hg.), Handbook of Direct Democracy in Central and Eastern Europe after 1989 (Opladen-Berlin-Toronto 2018).

Wilhelm MommSEN (Hg.), Deutsche Parteiprogramme (= Deutsches Handbuch der Politik 1, München 1964).

Gábor PAJKOSSY (Hg.), Magyarország története a 19. században. Szöveggyüjtemény (Budapest 2003).
Reinhard SCHIFFERS, Elemente direkter Demokratie im Weimarer Regierungssystem (Düsseldorf 1971).

DERS., Schlechte Weimarer Erfahrungen? in: Hans Herbert von ARNIM (Hg.), Direkte Demokratie. Beiträge auf dem 3. Speyerer Demokratieforum vom 27. bis 29. Oktober 1999 an der Deutschen Hochschule für Verwaltungswissenschaften (= Schriftenreihe der Hochschule Speyer 140, Berlin 2000) 51-65.

László SzALAY, A' schweitzi diéta 's a' foederatívrendszer, in: Pesti Hírlap Nr. 368 v. 11. 7. 1844, Nachruck: DERS. (Hg.), Publicistai dolgozatok, Bd. 1: 202-205 (Pest 1847).

Adrian VATTER, Direkte Demokratie in der Schweiz, in: Markus Freitag, Uwe Wagschal (Hgg.), Direkte Demokratie. Bestandaufnahmen und Wirkungen im internationalen Vergleich (= PolicyForschung und Vergleichende Regierungslehre 3, Berlin 2007) 71-113.

DERS., Kantonale Demokratien im Vergleich. Entstehungsgründe, Interaktionen und Wirkungen politischer Institutionen in der Schweizer Kantonen (Opladen 2002).

Hugó VeigelsberG, A jövendő törvényhozás. Adalék a parlamentarizmus kérdéséhez, in: Huszadik Század 4 (1903/7) 1-17. 